\title{
NEW DETERMINANTS AND THE CAYLEY-HAMILTON THEOREM FOR MATRICES OVER LIE NILPOTENT RINGS
}

\author{
JENŐ SZIGETI
}

(Communicated by Lance W. Small)

\begin{abstract}
We construct the so-called right adjoint sequence of an $n \times n$ matrix over an arbitrary ring. For an integer $m \geq 1$ the right $m$-adjoint and the right $m$-determinant of a matrix is defined by the use of this sequence. Over $m$-Lie nilpotent rings a considerable part of the classical determinant theory, including the Cayley-Hamilton theorem, can be reformulated for our right adjoints and determinants. The new theory is then applied to derive the PI of algebraicity for matrices over the Grassmann algebra.
\end{abstract}

\section{INTRODUCTION}

The main aim of the present paper is to develop a new theory of determinants for $n \times n$ matrices over rings satisfying the polynomial identity of $m$-Lie nilpotency:

$$
\left[\left[\left[\ldots\left[\left[x_{1}, x_{2}\right], x_{3}\right], \ldots\right], x_{m}\right], x_{m+1}\right]=0 .
$$

Our treatment is based on the construction of the right adjoint sequence of a matrix. Over $m$-Lie nilpotent rings the right (left) multiplication of a matrix by its right (left) $m$-adjoint will result in a scalar multiple of the unit matrix. This scalar coincides with the right (left) $m$-determinant of the given matrix. The above mentioned property of our adjoints heavily depends on the PI-condition imposed on the base ring. By far the most important example of such a base ring is the Grassmann algebra, which is 2-Lie nilpotent. A complete description of Lie nilpotent group rings can be found in [11]; see also [4]. Since all identities under consideration are inherited by polynomial rings of commuting indeterminates over the base ring, it will be possible to use the adjoints of matrices over polynomial rings. The theory (at present stage) culminates in the non-commutative analogue of the classical Cayley-Hamilton theorem. In the last section we apply this theorem to derive the polynomial identity of "algebraicity" for matrices over the infinite dimensional Grassmann algebra. This identity is one of the few explicitly known identities of the $n \times n$ matrix ring over the Grassmann algebra (see [1]). Combining our result with some theorems of Kemer $([7,8,9])$, we obtain the remarkable fact that any T-ideal contains a "polynomial of algebraicity". It is also clear from works due to Berele, Kemer and others, that the T-ideal of the identities of the $n \times n$ matrix ring over an infinite dimensional Grassmann algebra plays an exceptional role in

Received by the editors December 19, 1995 and, in revised form, March 6, 1996.

1991 Mathematics Subject Classification. Primary 16A38, 15A15; Secondary 15A33.

Supported by OTKA of Hungary, grant no. T7558, and by the Computer and Automation Institute of the Hungarian Academy of Science.

(C)1997 American Mathematical Society 
the study of T-ideals (see also [2,3] and [12]). Any result concerning the identities of algebras, with a verbally prime T-ideal of identities, seems to be of interest for PI-theory.

The present theory of determinants also serves as an efficient tool in solving systems of linear equations over Lie nilpotent rings. In a forthcoming paper the author will provide a detailed study of such systems of linear equations.

\section{SUbRingS IN LiE NILPOTENT RINGS}

Let $\left\{x_{1}, x_{2}, \ldots, x_{m}, \ldots\right\}$ be a set of non-commuting indeterminates and define the sequence $\left(f_{m}\left(x_{1}, x_{2}, \ldots, x_{m}\right)\right)$ of polynomials in $\mathbf{Z}\left\langle x_{1}, x_{2}, \ldots, x_{m}, \ldots\right\rangle$ by the following recursion: $f_{1}\left(x_{1}\right)=x_{1}$ and for $m \geq 1$ let

$$
f_{m+1}\left(x_{1}, x_{2}, \ldots, x_{m}, x_{m+1}\right)=\left[f_{m}\left(x_{1}, x_{2}, \ldots, x_{m}\right), x_{m+1}\right] .
$$

A ring $R$ is called $m$-Lie nilpotent if $f_{m+1}\left(x_{1}, x_{2}, \ldots, x_{m}, x_{m+1}\right)=0$ is a polynomial identity in $R$. Clearly, 1-Lie nilpotency coincides with commutativity and as is well known (see [10]) the Grassmann algebra is 2-Lie nilpotent. For a $m$-Lie nilpotent ring $R$ define the subring $R^{(m)} \leq R$ as follows:

$$
R^{(m)}=\left\{r \in R \mid f_{m}\left(r, s_{1}, s_{2}, \ldots, s_{m-1}\right)=0 \text { for all } s_{1}, s_{2}, \ldots, s_{m-1} \in R\right\} .
$$

The fact that $R^{(m)}$ is a subring is a consequence of $[u v, s]=[u, v s]+[v, s u]$.

For us let $[R, R]$ denote the additive subgroup of $R$ generated by the set $\{[u, v] \mid$ $u, v \in R\} \subseteq R$ of commutator elements. Note that $[R, R]$ consists of finite sums of commutators and in general is not an ideal of $R$. We shall make use of the following inclusion: $[R, R] \subseteq R^{(m)}$, i.e. that $[u, v] \in R^{(m)}$ for all $u, v \in R$. Indeed, the above inclusion is a consequence of

$$
f_{m}\left([u, v], s_{1}, s_{2}, \ldots, s_{m-1}\right)=f_{m+1}\left(u, v, s_{1}, s_{2}, \ldots, s_{m-1}\right) .
$$

It is obvious that $R^{(m)}$ itself is a $(m-1)$-Lie nilpotent ring. A stronger statement is the following.

Lemma 2.1. Let $R$ be a $m$-Lie nilpotent ring with $m \geq 2$ and let $b \in R$ be an arbitrary element. Then the subring $\left\langle R^{(m)}, b\right\rangle \leq R$ generated by the set $R^{(m)} \cup\{b\} \subseteq$ $R$ is $(m-1)$-Lie nilpotent.

Proof. We shall make repeated use of the following identities:

$$
\begin{gathered}
{[v, u]=-[u, v],} \\
{[u v, s]=u[v, s]+[u, s] v,\left[u b^{i}, b^{j}\right]=\left[u, b^{j}\right] b^{i},} \\
{[[u, v], r]+[[v, r], u]+[[r, u], v]=0 .}
\end{gathered}
$$

First we claim that $\left[\left[\left[\ldots\left[\left[u_{1}, u_{2}\right], u_{3}\right], \ldots\right], u_{k}\right], r\right]$ can be written as a sum of terms of the form

$$
\pm\left[\left[\ldots\left[\left[r, u_{\alpha(1)}\right], u_{\alpha(2)}\right], \ldots\right], u_{\alpha(k)}\right], \alpha \in \operatorname{Sym}(\{1,2, \ldots, k\}) .
$$

We proceed by induction. If $k=1$ then $\left[u_{1}, r\right]=-\left[r, u_{1}\right]$. Assume now that the claim holds for an integer $k \geq 1$. Using $[v, u]=-[u, v]$ and the Jacobi identity, we obtain that

$$
\begin{gathered}
{\left[\left[\left[\left[\ldots\left[\left[u_{1}, u_{2}\right], u_{3}\right], \ldots\right], u_{k}\right], u_{k+1}\right], r\right]=} \\
{\left[\left[\left[\left[\ldots\left[\left[u_{1}, u_{2}\right], u_{3}\right], \ldots\right], u_{k}\right], r\right], u_{k+1}\right]-\left[\left[\left[\ldots\left[\left[u_{1}, u_{2}\right], u_{3}\right], \ldots\right], u_{k}\right],\left[r, u_{k+1}\right]\right] .}
\end{gathered}
$$

On applying the induction hypothesis to

$$
\left[\left[\left[\ldots\left[\left[u_{1}, u_{2}\right], u_{3}\right], \ldots\right], u_{k}\right], r\right] \text { and to } \quad\left[\left[\left[\ldots\left[\left[u_{1}, u_{2}\right], u_{3}\right], \ldots\right], u_{k}\right], r^{\prime}\right]
$$


with $r^{\prime}=\left[r, u_{k+1}\right]$, we get the desired sum presentation of

$$
\left[\left[\left[\left[\ldots\left[\left[u_{1}, u_{2}\right], u_{3}\right], \ldots\right], u_{k}\right], u_{k+1}\right], r\right] .
$$

It follows that $\left[\left[\ldots\left[\left[\left[\left[\ldots\left[u_{1}, u_{2}\right], \ldots\right], u_{k}\right], r\right], u_{k+1}\right], \ldots\right], u_{m-1}\right]$ can be written as a sum of terms of the form

$$
\pm\left[\left[\ldots\left[\left[\left[\ldots\left[\left[r, u_{\alpha(1)}\right], u_{\alpha(2)}\right], \ldots\right], u_{\alpha(k)}\right], u_{k+1}\right], \ldots\right], u_{m-1}\right], \alpha \in \operatorname{Sym}(\{1,2, \ldots, k\}) .
$$

Thus we have

$$
r \in R^{(m)} \Rightarrow\left[\left[\ldots\left[\left[\left[\left[\ldots\left[u_{1}, u_{2}\right], \ldots\right], u_{k}\right], r\right], u_{k+1}\right], \ldots\right], u_{m-1}\right]=0
$$

for all $u_{1}, u_{2}, \ldots, u_{m-1} \in R$.

Since $b r=[b, r]+r b$ and $[b, r] \in[R, R] \subseteq R^{(m)}$, we obtain that each element $x \in\left\langle R^{(m)}, b\right\rangle$ can be written as $x=r_{0}+r_{1} b+\ldots+r_{d} b^{d}$ with $r_{0}, r_{1}, \ldots, r_{d} \in$ $R^{(m)}$. In order to check that $\left[\left[\ldots\left[\left[x_{1}, x_{2}\right], x_{3}\right], \ldots\right], x_{m}\right]=0$ is a polynomial identity on $\left\langle R^{(m)}, b\right\rangle$, it is enough to consider substitutions of the form $x_{1}=r_{1} b^{i_{1}}$, $x_{2}=r_{2} b^{i_{2}}, \ldots, x_{m}=r_{m} b^{i_{m}}$ with $r_{1}, r_{2}, \ldots, r_{m} \in R^{(m)}$. For $1 \leq k \leq m$ put $w_{k}=\left[\left[\ldots\left[\left[r_{1} b^{i_{1}}, r_{2} b^{i_{2}}\right], r_{3} b^{i_{3}}\right], \ldots\right], r_{k} b^{i_{k}}\right]\left(w_{1}=r_{1} b^{i_{1}}\right)$ then we have

$$
\left[w_{m-1}, r_{m} b^{i_{m}}\right]=r_{m}\left[w_{m-1}, b^{i_{m}}\right]+\left[w_{m-1}, r_{m}\right] b^{i_{m}}
$$

together with

$$
r_{m} \in R^{(m)} \Rightarrow\left[w_{m-1}, r_{m}\right]=\left[\left[\left[\ldots\left[\left[r_{1} b^{i_{1}}, r_{2} b^{i_{2}}\right], r_{3} b^{i_{3}}\right], \ldots\right], r_{m-1} b^{i_{m-1}}\right], r_{m}\right]=0,
$$

whence we obtain that

$$
\left[\left[\ldots\left[\left[x_{1}, x_{2}\right], x_{3}\right], \ldots\right], x_{m}\right]=r_{m}\left[w_{m-1}, b^{i_{m}}\right] .
$$

Assume now that

$$
\left[\left[\ldots\left[\left[x_{1}, x_{2}\right], x_{3}\right], \ldots\right], x_{m}\right]=r_{m}\left[r_{m-1}\left[r_{m-2}\left[\ldots\left[r_{k+1}\left[w_{k}, b^{i_{k+1}}\right], b^{i_{k+2}}\right], \ldots\right], b^{i_{m-1}}\right], b^{i_{m}}\right]
$$

for some $2 \leq k \leq m-1$. Since

$$
w_{k}=\left[w_{k-1}, r_{k} b^{i_{k}}\right]=r_{k}\left[w_{k-1}, b^{i_{k}}\right]+\left[w_{k-1}, r_{k}\right] b^{i_{k}},
$$

we obtain that

$$
\begin{aligned}
& {\left[\left[\ldots\left[\left[x_{1}, x_{2}\right], x_{3}\right], \ldots\right], x_{m}\right]} \\
& \quad=r_{m}\left[r_{m-1}\left[r_{m-2}\left[\ldots\left[r_{k+1}\left[r_{k}\left[w_{k-1}, b^{i_{k}}\right], b^{i_{k+1}}\right], b^{i_{k+2}}\right], \ldots\right], b^{i_{m-1}}\right], b^{i_{m}}\right] \\
& \quad+r_{m}\left[r_{m-1}\left[r_{m-2}\left[\ldots\left[r_{k+1}\left[\left[w_{k-1}, r_{k}\right], b^{i_{k+1}}\right], b^{i_{k+2}}\right], \ldots\right], b^{i_{m-1}}\right], b^{i_{m}}\right] b^{i_{k}} .
\end{aligned}
$$

Substitute $i_{k}=0$ in the above equation and use $\left[w_{k-1}, 1\right]=0$ together with

$$
r_{k} \in R^{(m)} \Rightarrow\left[\left[\ldots\left[\left[\left[\left[\ldots\left[r_{1} b^{i_{1}}, r_{2} b^{i_{2}}\right], \ldots\right], r_{k-1} b^{i_{k-1}}\right], r_{k}\right], r_{k+1} b^{i_{k+1}}\right], \ldots\right], r_{m} b^{i_{m}}\right]=0
$$

to derive

$$
r_{m}\left[r_{m-1}\left[r_{m-2}\left[\ldots\left[r_{k+1}\left[\left[w_{k-1}, r_{k}\right], b^{i_{k+1}}\right], b^{i_{k+2}}\right], \ldots\right], b^{i_{m-1}}\right], b^{i_{m}}\right]=0 .
$$

It follows that

$$
\begin{aligned}
& {\left[\left[\ldots\left[\left[x_{1}, x_{2}\right], x_{3}\right], \ldots\right], x_{m}\right]} \\
& \quad=r_{m}\left[r_{m-1}\left[r_{m-2}\left[\ldots\left[r_{k+1}\left[r_{k}\left[w_{k-1}, b^{i_{k}}\right], b^{i_{k+1}}\right], b^{i_{k+2}}\right], \ldots\right], b^{i_{m-1}}\right], b^{i_{m}}\right] .
\end{aligned}
$$

On repeating the above step, finally we get that

$$
\left[\left[\ldots\left[\left[x_{1}, x_{2}\right], x_{3}\right], \ldots\right], x_{m}\right]=r_{m}\left[r_{m-1}\left[\ldots\left[r_{3}\left[r_{2}\left[w_{1}, b^{i_{2}}\right], b^{i_{3}}\right], b^{i_{4}}\right], \ldots\right], b^{i_{m}}\right],
$$

i.e. that

$$
\left[\left[\ldots\left[\left[r_{1} b^{i_{1}}, r_{2} b^{i_{2}}\right], r_{3} b^{i_{3}}\right], \ldots\right], r_{m} b^{i_{m}}\right]=r_{m}\left[r_{m-1}\left[\ldots\left[r_{3}\left[r_{2}\left[r_{1}, b^{i_{2}}\right], b^{i_{3}}\right], b^{i_{4}}\right], \ldots\right], b^{i_{m}}\right] b^{i_{1}} .
$$


To conclude the proof, substitute $i_{1}=0$ in the last equation and use

$$
r_{1} \in R^{(m)} \Rightarrow\left[\left[\ldots\left[\left[r_{1}, r_{2} b^{i_{2}}\right], r_{3} b^{i_{3}}\right], \ldots\right], r_{m} b^{i_{m}}\right]=0
$$

to derive

$$
r_{m}\left[r_{m-1}\left[\ldots\left[r_{3}\left[r_{2}\left[r_{1}, b^{i_{2}}\right], b^{i_{3}}\right], b^{i_{4}}\right], \ldots\right], b^{i_{m}}\right]=0 .
$$

\section{ThE RIGHT ADJOINTS AND THE RIGHT DETERMINANTS OF $n \times n$ MATRICES}

First we define the preadjoint of a $n \times n$ matrix $A \in M_{n}(R)$ over an arbitrary ring $R$ with 1 . Let

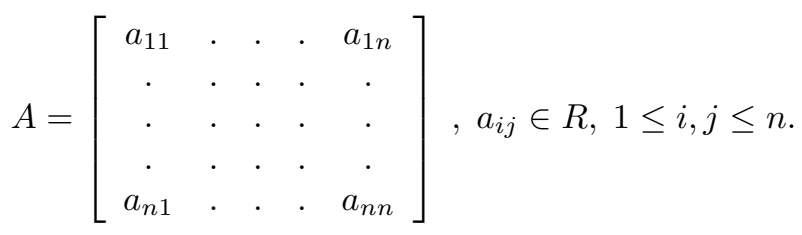

For the permutations $\tau \in \operatorname{Sym}(\{1, \ldots, s-1, s+1, \ldots, n\})$ and $\rho \in \operatorname{Sym}(\{1,2, \ldots, n\})$ we shall make use of the following product:

$$
a(s, \tau, \rho)=a_{\tau(1) \rho(\tau(1))} \ldots a_{\tau(s-1) \rho(\tau(s-1))} a_{\tau(s+1) \rho(\tau(s+1))} \ldots a_{\tau(n) \rho(\tau(n))} .
$$

For the integers $1 \leq r, s \leq n$ let $\Pi(s, r)$ denote the following set of $\{1,2, \ldots, n\} \rightarrow$ $\{1,2, \ldots, n\}$ permutations: $\Pi(s, r)=\{\rho \in \operatorname{Sym}(\{1,2, \ldots, n\}) \mid \rho(s)=r\}$.

The (two sided) preadjoint of $A$ is the matrix $A^{*}=\left[a_{r s}^{*}\right] \in M_{n}(R)$, where

$$
a_{r s}^{*}=\sum \operatorname{sgn}(\rho) a(s, \tau, \rho), 1 \leq r, s \leq n,
$$

and the sum is taken over all permutations $\tau \in \operatorname{Sym}(\{1, \ldots, s-1, s+1, \ldots, n\})$ and $\rho \in \Pi(s, r)$. We note that if $R$ is commutative then the ordinary adjoint of $A$ is $\operatorname{adj}(A)=\left[ \pm \bar{a}_{r s}\right]$, where $\bar{a}_{r s}$ is an $(n-1) \times(n-1)$ determinant, a signed sum of products, each of length $n-1$. In the above definition of $a_{r s}^{*}$ each such product is taken in all its $(n-1)$ ! possible rearrangements. Thus we have

$$
A^{*}=(n-1) ! \operatorname{adj}(A)
$$

in case of a commutative ring $R$. Our development is based on the following crucial result.

Theorem 3.1. The product matrix $A A^{*} \in M_{n}(R)$ can be written in the following form:

$$
A A^{*}=b E+C,
$$

where $b \in R$ is the $(1,1)$ entry in $A A^{*}, E \in M_{n}(R)$ is the unit matrix and $C=\left[c_{i j}\right]$ with $c_{11}=0$ and each $c_{i j} \in R, 1 \leq i, j \leq n$, is a sum of commutators, i.e. a sum of elements of the form $[u, v](u, v \in R)$.

Proof. Let $A A^{*}=\left[b_{i j}\right]$ and take $b=b_{11}$; then it is enough to show that each $b_{i j}, i \neq j$, and each $b_{i i}-b_{11}, i \neq 1$ is a sum of commutators. Now

$$
b_{i j}=\sum_{r=1}^{n} a_{i r} a_{r j}^{*}=\sum_{(\tau, \rho) \in \mathcal{I}(j)} \operatorname{sgn}(\rho) a_{i \rho(j)} a(j, \tau, \rho),
$$


where $\mathcal{I}(j)$ denotes the set of all ordered pairs $(\tau, \rho)$ with $\tau \in \operatorname{Sym}(\{1, \ldots, j-1$, $j+1, \ldots, n\})$ and $\rho \in \operatorname{Sym}(\{1,2, \ldots, n\})$. For $i \neq j$ define a function $I_{i j}: \mathcal{I}(j) \rightarrow$ $\mathcal{I}(j)$ as follows:

$$
I_{i j}(\tau, \rho)=\left(\tau^{\prime}, \rho^{\prime}\right)
$$

where

$\left(\tau^{\prime}(1), \ldots, \tau^{\prime}(j-1), \tau^{\prime}(j+1), \ldots, \tau^{\prime}(n)\right)=(\tau(k+1), \ldots, \tau(n), \tau(k), \tau(1), \ldots, \tau(k-1))$ as ordered $(n-1)$-tuples with $k=\tau^{-1}(i)$ and

$$
\rho^{\prime}(t)=\left\{\begin{array}{l}
\rho(t) \text { if } t \notin\{i, j\} \\
\rho(i) \text { if } t=j \\
\rho(j) \text { if } t=i
\end{array}\right.
$$

Clearly, $\operatorname{sgn}\left(\rho^{\prime}\right)=-\operatorname{sgn}(\rho)$, moreover we have $(\mathrm{C})$ :

$$
\begin{gathered}
\operatorname{sgn}(\rho) a_{i \rho(j)} a(j, \tau, \rho)+\operatorname{sgn}\left(\rho^{\prime}\right) a_{i \rho^{\prime}(j)} a\left(j, \tau^{\prime}, \rho^{\prime}\right) \\
=\operatorname{sgn}(\rho)\left[a_{i \rho(j)} a_{\tau(1) \rho(\tau(1))} \ldots a_{\tau(k-1) \rho(\tau(k-1))}, a_{\tau(k) \rho(\tau(k))} \ldots a_{\tau(n) \rho(\tau(n))}\right] .
\end{gathered}
$$

A straightforward reasoning shows that

$$
I_{i j}\left(I_{i j}(\tau, \rho)\right)=(\tau, \rho)
$$

for all $(\tau, \rho) \in \mathcal{I}(j)$. Thus $I_{i j}$ is a permutation of $\mathcal{I}(j)$ and $\mathcal{I}(j)$ is a union of pairwise disjoint two element cycles of $I_{i j}$. It follows that $b_{i j}$ is a sum of commutators of the form $(\mathrm{C})$.

For $i \geq 2$ we have

$$
b_{i i}-b_{11}=\sum_{(\tau, \rho) \in \mathcal{I}(i)} \operatorname{sgn}(\rho) a_{i \rho(i)} a(i, \tau, \rho)-\sum_{(\varepsilon, \pi) \in \mathcal{I}(1)} \operatorname{sgn}(\pi) a_{1 \pi(1)} a(1, \varepsilon, \pi) .
$$

Define the functions $I_{i}: \mathcal{I}(i) \rightarrow \mathcal{I}(1)$ and $J_{i}: \mathcal{I}(1) \rightarrow \mathcal{I}(i)$ as follows:

$$
I_{i}(\tau, \rho)=\left(\varepsilon_{\tau}, \rho\right),
$$

where

$$
\left(\varepsilon_{\tau}(2), \ldots, \varepsilon_{\tau}(n)\right)=(\tau(k+1), \ldots, \tau(n), i, \tau(1), \ldots, \tau(k-1))
$$

as ordered $(n-1)$-tuples with $k=\tau^{-1}(1)$;

$$
J_{i}(\varepsilon, \pi)=\left(\tau_{\varepsilon}, \pi\right),
$$

where

$$
\left(\tau_{\varepsilon}(1), \ldots, \tau_{\varepsilon}(i-1), \tau_{\varepsilon}(i+1), \ldots, \tau_{\varepsilon}(n)\right)=(\varepsilon(l+1), \ldots, \varepsilon(n), 1, \varepsilon(2), \ldots, \varepsilon(l-1))
$$

as ordered $(n-1)$-tuples with $l=\varepsilon^{-1}(i)$.

It is easy to see that $I_{i}$ and $J_{i}$ are mutual inverses of each other. Since we have $(\mathrm{CC})$ :

$$
\begin{gathered}
\operatorname{sgn}(\rho) a_{i \rho(i)} a(i, \tau, \rho)-\operatorname{sgn}(\rho) a_{1 \rho(1)} a\left(1, \varepsilon_{\tau}, \rho\right) \\
=\operatorname{sgn}(\rho)\left[a_{i \rho(i)} a_{\tau(1) \rho(\tau(1))} \ldots a_{\tau(k-1) \rho(\tau(k-1))}, a_{\tau(k) \rho(\tau(k))} \ldots a_{\tau(n) \rho(\tau(n))}\right],
\end{gathered}
$$

$b_{i i}-b_{11}$ is a sum of commutators of the form (CC).

We define the right adjoint sequence $\left(A_{k}\right)_{k \geq 1}$ of a matrix $A \in M_{n}(R)$, over an arbitrary ring $R$, by the following recursion: $A_{1}=A^{*}$ and for $k \geq 1$ let

$$
A_{k+1}=\left(A A_{1} \ldots A_{k}\right)^{*} \text {. }
$$

Thus $\left(A_{k}\right)_{k \geq 1}$ is an infinite sequence of matrices in $M_{n}(R)$. For an integer $m \geq 1$ the

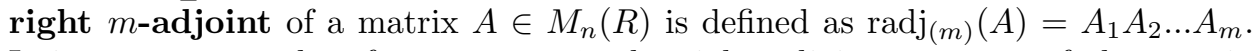
It is easy to see that for any $i \geq 1$, the right adjoint sequence of the matrix 
$B=A \operatorname{radj}_{(i)}(A)=A A_{1} \ldots A_{i}$ is exactly $\left(A_{k}\right)_{k \geq i+1}$. The right $m$-determinant $\operatorname{rdet}_{(m)}(A) \in R$ of $A \in M_{n}(R)$ is defined as the $(1,1)$ entry of the product matrix $A \operatorname{radj}_{(m)}(A)=A A_{1} \ldots A_{m}$. The observation on the right adjoint sequence of $B$ immediately gives that for $i, j \geq 1$ we have

$$
\operatorname{radj}_{(i+j)}(A)=\operatorname{radj}_{(i)}(A) \operatorname{radj}_{(j)}\left(A A_{1} \ldots A_{i}\right), \operatorname{rdet}_{(i+j)}(A)=\operatorname{rdet}_{(j)}\left(A A_{1} \ldots A_{i}\right) .
$$

We note that the right $m$-determinant of a $n \times n$ matrix $A \in M_{n}(R)$ over a commutative ring $R$ is

$$
\operatorname{rdet}_{(m)}(A)=[(n-1) !]^{1+n+n^{2}+\ldots+n^{m-1}}(\operatorname{det}(A))^{n^{m-1}} .
$$

This can be shown by using

$$
A A_{1}=(n-1) ! A \operatorname{adj}(A)=(n-1) ! \operatorname{det}(A) E, \quad \operatorname{rdet}_{(m)}(A)=\operatorname{rdet}_{(m-1)}\left(A A_{1}\right)
$$

and applying a straightforward induction.

Theorem 3.2. Let $A \in M_{n}(R)$, where $R$ is a $m$-Lie nilpotent ring with 1 . Then

$$
A \operatorname{radj}_{(m)}(A)=\operatorname{rdet}_{(m)}(A) E,
$$

where $E \in M_{n}(R)$ is the unit matrix.

Proof. If $m=1$ then $\operatorname{radj}_{(1)}(A)=A_{1}=A^{*}$ and Theorem 3.1 give that

$$
A \operatorname{radj}_{(1)}(A)=\operatorname{rdet}_{(1)}(A) E+C,
$$

where $C=0$ follows from the fact that $[R, R]=\{0\}$ for the 1-Lie nilpotent (commutative) ring $R$. Assume now that for an integer $m \geq 2$ the statement of the theorem holds for $m-1$. Take $A \in M_{n}(R)$ with $R$ being $m$-Lie nilpotent; then Theorem 3.1 together with $[R, R] \subseteq R^{(m)}$ gives that $A A_{1}=b_{1} E+C_{1}$, where $b_{1}=\operatorname{rdet}_{(1)}(A)$ and $C_{1} \in M_{n}\left(R^{(m)}\right)$. Form the subring $R_{1}=\left\langle R^{(m)}, b_{1}\right\rangle \leq R$ generated by the subset $R^{(m)} \cup\left\{b_{1}\right\} \subseteq R$. Lemma 2.1 ensures that $R_{1}$ is $(m-1)$-Lie nilpotent. Since $A A_{1} \in M_{n}\left(R_{1}\right)$, our inductive hypothesis gives that

$$
A A_{1} \operatorname{radj}_{(m-1)}\left(A A_{1}\right)=\operatorname{rdet}_{(m-1)}\left(A A_{1}\right) E .
$$

In view of $\operatorname{radj}_{(m)}(A)=\operatorname{radj}_{(1)}(A) \operatorname{radj}_{(m-1)}\left(A A_{1}\right)=A_{1} \operatorname{radj}_{(m-1)}\left(A A_{1}\right)$ and $\operatorname{rdet}_{(m)}(A)=\operatorname{rdet}_{(m-1)}\left(A A_{1}\right)$ the theorem is proved.

In the proof of Theorem 3.2 we have $A A_{1} \in M_{n}\left(R_{1}\right)$, where $R_{1}$ is a $(m-1)$ Lie nilpotent subring of $R$. If $m=2$ then the commutativity of $R_{1}$ implies that $\operatorname{rdet}_{(2)}(A)=\operatorname{rdet}_{(1)}\left(A A_{1}\right)=(n-1) ! \operatorname{det}\left(A A_{1}\right)$. Using the fact that $A_{1}=A^{*}$ we can derive an explicit formula for $\operatorname{rdet}_{(2)}(A)$.

Proposition 3.3. The right 2-determinant of a $n \times n$ matrix $A \in M_{n}(R)$ over a 2-Lie nilpotent ring $R$ is the following:

$$
\operatorname{rdet}_{(2)}(A)=(n-1) ! \sum \operatorname{sgn}(\alpha) \operatorname{sgn}\left(\rho_{1}\right) \ldots \operatorname{sgn}\left(\rho_{n}\right) a(\alpha, \overline{\boldsymbol{\tau}}, \overline{\boldsymbol{\rho}}),
$$

where

$$
a(\alpha, \overline{\boldsymbol{\tau}}, \overline{\boldsymbol{\rho}})=a_{1 \rho_{1}(\alpha(1))} a\left(\alpha(1), \tau_{1}, \rho_{1}\right) \ldots a_{n \rho_{n}(\alpha(n))} a\left(\alpha(n), \tau_{n}, \rho_{n}\right)
$$

and the summation runs over all ordered $(2 n+1)$-tuples $\left(\alpha, \tau_{1}, \ldots, \tau_{n}, \rho_{1}, \ldots, \rho_{n}\right)$ of permutations with $\alpha, \rho_{i} \in \operatorname{Sym}(\{1,2, \ldots, n\})$ and $\tau_{i} \in \operatorname{Sym}(\{1, \ldots, \alpha(i)-1, \alpha(i)+$ $1, \ldots, n\}$ ) for all $1 \leq i \leq n$. 
Proof. We use the notations and the calculations of the proof of Theorem 3.1. By the definition of the ordinary determinant, we get that

$$
\operatorname{det}\left(A A^{*}\right)=\sum_{\alpha \in \operatorname{Sym}(\{1, \ldots, n\})} \operatorname{sgn}(\alpha) b_{1 \alpha(1)} b_{2 \alpha(2)} \ldots b_{n \alpha(n)}
$$

and here

$$
b_{i \alpha(i)}=\sum_{(\tau, \rho) \in \mathcal{I}(\alpha(i))} \operatorname{sgn}(\rho) a_{i \rho(\alpha(i))} a(\alpha(i), \tau, \rho)
$$

with $\tau \in \operatorname{Sym}(\{1, \ldots, \alpha(i)-1, \alpha(i)+1, \ldots, n\})$ and $\rho \in \operatorname{Sym}(\{1,2, \ldots, n\})$. On multiplying the above sums for $1 \leq i \leq n$, we obtain the required formula.

Example. The right 2-determinant of a $2 \times 2$ matrix

$$
A=\left[\begin{array}{ll}
a_{11} & a_{12} \\
a_{21} & a_{22}
\end{array}\right], a_{i j} \in R, 1 \leq i, j \leq 2
$$

over a 2-Lie nilpotent ring $R$ is

$$
\operatorname{rdet}_{(2)}(A)=\operatorname{det}\left(A A^{*}\right)=\left(a_{11} a_{22}-a_{12} a_{21}\right)\left(a_{22} a_{11}-a_{21} a_{12}\right)-\left[a_{12}, a_{11}\right]\left[a_{21}, a_{22}\right] .
$$

\section{The Cayley-Hamilton theorem}

Let $R[x]$ denote the ring of polynomials of the single commuting indeterminate $x$, with coefficients in $R$. It is easy to see that any matrix $A(x) \in M_{n}(R[x])$ can be uniquely written in the following form:

$$
A(x)=A_{0}+A_{1} x+\ldots+A_{d} x^{d} \text { with } A_{0}, A_{1}, \ldots, A_{d} \in M_{n}(R) .
$$

Thus we get a natural isomorphism of rings $M_{n}(R[x]) \cong M_{n}(R)[x]$. We define the right $m$-characteristic polynomial of a matrix $A \in M_{n}(R)$ as the right $m$-determinant of the matrix $A-E x \in M_{n}(R[x])$, where $E \in M_{n}(R)$ is the unit matrix:

$p(x)=\lambda_{0}+\lambda_{1} x+\ldots+\lambda_{d} x^{d}=\operatorname{rdet}_{(m)}(A-E x) \in R[x], \lambda_{0}, \lambda_{1}, \ldots, \lambda_{d} \in R, \lambda_{d} \neq 0$.

It is not hard to see that $\lambda_{0}=\operatorname{rdet}_{(m)}(A)$.

Proposition 4.1. If $p(x)=\lambda_{0}+\lambda_{1} x+\ldots+\lambda_{d} x^{d}$ is the right m-characteristic polynomial of a $n \times n$ matrix $A \in M_{n}(R)$ then $d=n^{m}$ and

$$
\lambda_{d}=(-1)^{n}[(n-1) !]^{1+n+n^{2}+\ldots+n^{m-1}} .
$$

Proof. Let $\left((A-E x)_{k}\right)_{k>1}$ be the right adjoint sequence of the matrix $A-E x$. We claim that in $(A-E x)(A-E x)_{1} \ldots(A-E x)_{m}$ the leading monomial of each diagonal entry is $(-1)^{n}[(n-1) !]^{1+n+n^{2}+\ldots+n^{m-1}} x^{n^{m}}$ and the degree of each non diagonal entry is less than $n^{m}$. We apply an induction on $m$. If $m=1$ then a direct computation of the entries of $(A-E x)(A-E x)^{*}$ can prove the claim (an argument similar to the following one on $T(x)(T(x))^{*}$ also works). Assume now that our claim holds for $m-1$. Put

$$
T(x)=\left[t_{i j}(x)\right]=(A-E x)(A-E x)_{1} \ldots(A-E x)_{m-1} ;
$$

then $(A-E x)_{m}=(T(x))^{*}$ and consider the matrix

$$
\left[b_{i j}(x)\right]=T(x)(T(x))^{*}=(A-E x)(A-E x)_{1} \ldots(A-E x)_{m} .
$$


Using the calculations of the proof of Theorem 3.1, we get that

$$
b_{i j}(x)=\sum_{(\tau, \rho) \in \mathcal{I}(j)} \operatorname{sgn}(\rho) t_{i \rho(j)}(x) t(j, \tau, \rho)
$$

with $t(j, \tau, \rho)=t_{\tau(1) \rho(\tau(1))}(x) \ldots t_{\tau(j-1) \rho(\tau(j-1))}(x) t_{\tau(j+1) \rho(\tau(j+1))}(x) \ldots t_{\tau(n) \rho(\tau(n))}(x)$.

If $(\tau, \rho) \in \mathcal{I}(j)$ and $i \neq j$ then it is easy to see that at least one of the terms

$$
t_{i \rho(j)}(x), t_{\tau(1) \rho(\tau(1))}(x), \ldots, t_{\tau(j-1) \rho(\tau(j-1))}(x), t_{\tau(j+1) \rho(\tau(j+1))}(x), \ldots, t_{\tau(n) \rho(\tau(n))}(x)
$$

is a non diagonal entry of $T(x)$. In view of our assumption on $T(x)$, we get that

$$
\operatorname{deg}\left(b_{i j}(x)\right) \leq n n^{m-1}-1<n^{m} .
$$

If $i=j$ then the assumption on $T(x)$ implies that all the summands of highest degree in (e) belong to $\rho=\mathrm{id}$. It follows that the leading monomial of $b_{i i}(x)$ coincides with the leading monomial of

$$
\sum_{\tau \in \operatorname{Sym}(\{1, \ldots, i-1, i+1, \ldots, n\})} t_{i i}(x) t_{\tau(1) \tau(1)}(x) \ldots t_{\tau(i-1) \tau(i-1)}(x) t_{\tau(i+1) \tau(i+1)}(x) \ldots t_{\tau(n) \tau(n)}(x),
$$

which is

$$
\begin{gathered}
(n-1) !\left((-1)^{n}[(n-1) !]^{1+n+n^{2}+\ldots+n^{m-2}} x^{n^{m-1}}\right)^{n} \\
=(-1)^{n}[(n-1) !]^{1+n+n^{2}+\ldots+n^{m-1}} x^{n^{m}} \cdot \square
\end{gathered}
$$

The proof of the next theorem is similar to one of the classical proofs of the Cayley-Hamilton theorem.

Theorem 4.2. If $p(x)=\lambda_{0}+\lambda_{1} x+\ldots+\lambda_{d} x^{d}$ is the right m-characteristic polynomial of a $n \times n$ matrix $A \in M_{n}(R)$ over a m-Lie nilpotent ring $R$ then the left substitution of $A$ into $p(x)$ is zero: $(A) p=E \lambda_{0}+A \lambda_{1}+\ldots+A^{d} \lambda_{d}=0$.

Proof. The right $m$-adjoint of the matrix $A-E x$ can be written in the form:

$$
\operatorname{radj}_{(m)}(A-E x)=B_{0}+B_{1} x+\ldots+B_{t} x^{t}
$$

with $B_{0}, B_{1}, \ldots, B_{t} \in M_{n}(R)$. Since the $m$-Lie nilpotency of $R$ implies the $m$-Lie nilpotency of the polynomial ring $R[x]$, Theorem 3.2 gives that

$$
(A-E x)\left(B_{0}+B_{1} x+\ldots+B_{t} x^{t}\right)=p(x) E
$$

with $E$ being the unit matrix in $M_{n}(R[x])$. Now passing to $M_{n}(R)[x]$ and matching the coefficients of the powers of $x$ in the left and right sides of the above equation, we get the following sequence of equations:

$A B_{0}=E \lambda_{0}, A B_{1}-B_{0}=E \lambda_{1}, \ldots, A B_{t}-B_{t-1}=E \lambda_{t},-B_{t}=E \lambda_{t+1}, t+1=d$.

The left multiplication of $A B_{k}-B_{k-1}=E \lambda_{k}$ by $A^{k}$ gives that $A^{k+1} B_{k}-A^{k} B_{k-1}=$ $A^{k} \lambda_{k}$ and similarly $-B_{t}=E \lambda_{t+1} \Rightarrow-A^{t+1} B_{t}=A^{t+1} \lambda_{t+1}$. It follows that

$$
\begin{aligned}
E \lambda_{0} & +A \lambda_{1}+\ldots+A^{d} \lambda_{d} \\
& =A B_{0}+\left(A^{2} B_{1}-A B_{0}\right)+\ldots+\left(A^{t+1} B_{t}-A^{t} B_{t-1}\right)+\left(-A^{t+1} B_{t}\right)=0 .
\end{aligned}
$$

Remark 4.3. Using the same argument as in the proof of Theorem 4.2, we get that for any polynomial of the form $q(x)=p(x) h(x)$ with $p(x)=\operatorname{rdet}_{(m)}(A-E x)$, $h(x) \in R[x]$ the left substitution of $A$ into $q(x)$ is zero: $(A) q=0$. 
Example. For a $2 \times 2$ matrix $A \in M_{2}(R)$ over a 2 -Lie nilpotent ring $R$ we have

$$
\begin{gathered}
p(x)=\operatorname{rdet}_{(2)}(A-E x)=\operatorname{rdet}_{(2)}\left[\begin{array}{cc}
a_{11}-x & a_{12} \\
a_{21} & a_{22}-x
\end{array}\right] \\
=x^{4}-2\left(a_{11}+a_{22}\right) x^{3}+\left(a_{11}^{2}+a_{22}^{2}+2 a_{11} a_{22}+2 a_{22} a_{11}-a_{12} a_{21}-a_{21} a_{12}\right) x^{2} \\
+\left(\left(a_{11}+a_{22}\right)\left(a_{21} a_{12}-a_{22} a_{11}\right)+\left(a_{12} a_{21}-a_{11} a_{22}\right)\left(a_{11}+a_{22}\right)\right) x+\operatorname{rdet}_{(2)}(A) .
\end{gathered}
$$

Note that here we used the 2-Lie nilpotency of $R[x]$ and the example following Proposition 3.3. If we take

$$
A=\left[\begin{array}{ll}
1+v_{1} & v_{2} \\
3-v_{2} & v_{3}
\end{array}\right] \in M_{2}(G)
$$

where $G=\mathbf{Q}\left\langle v_{1}, v_{2}, \ldots\right\rangle$ is the Grassmann algebra, then $\operatorname{rdet}_{(2)}(A)=0$ and

$$
p(x)=x^{4}-2\left(1+v_{1}+v_{3}\right) x^{3}+\left(1+2 v_{1}-6 v_{2}+4 v_{3}\right) x^{2}+\left(6 v_{2}-2 v_{3}\right) x .
$$

\section{The identity of "Algebraicity" for matrices OVER THE GrassmanN ALGEBRA}

For the set $\left\{Y, Z, y_{1}, y_{2}, \ldots, y_{k}, \ldots\right\}$ of non-commuting indeterminates let $S_{k}\left(y_{1}, y_{2}, \ldots, y_{k}\right)$ denote the standard polynomial in $y_{1}, y_{2}, \ldots, y_{k}$ :

$$
S_{k}\left(y_{1}, y_{2}, \ldots, y_{k}\right)=\sum_{\alpha \in \operatorname{Sym}(\{1, \ldots, k\})} \operatorname{sgn}(\alpha) y_{\alpha(1)} y_{\alpha(2)} \ldots y_{\alpha(k)} .
$$

Theorem 5.1. $S_{2 n^{2}}\left(\left[Y^{2 n^{2}}, Z\right],\left[Y^{2 n^{2}-1}, Z\right], \ldots,\left[Y^{2}, Z\right],[Y, Z]\right)=0$ is a polynomial identity on the ring $M_{n}(G)$ of $n \times n$ matrices over the infinite dimensional Grassmann algebra $G=K\left\langle v_{1}, v_{2}, \ldots\right\rangle$ (here $K$ is a field of characteristic zero).

Proof. We write $G=G_{0}+G_{1}$, where $G_{0}$ is the subalgebra generated by the monomials in the $v_{i}$ 's of even length and $G_{1}$ is the subspace generated by the monomials in the $v_{i}$ 's of odd length. Clearly, the right 2-characteristic polynomial $p(x)=\operatorname{rdet}_{(2)}(Y-E x) \in G[x]$ of a matrix $Y \in M_{n}(G)$ can be uniquely written as $p(x)=p_{0}(x)+p_{1}(x)$ with $p_{0}(x) \in G_{0}[x]$ and $p_{1}(x) \in G_{1}[x]$. Since $G_{0}=Z(G)$ (the centre of $G$ ) and $G_{1} G_{1} \subseteq G_{0}$, we have

$$
q(x)=\left(p_{0}(x)+p_{1}(x)\right)\left(p_{0}(x)-p_{1}(x)\right)=\left(p_{0}(x)\right)^{2}-\left(p_{1}(x)\right)^{2} \in G_{0}[x] .
$$

The degree of $q(x)$ is $2 n^{2}$ and its leading coefficient is $[(n-1) !]^{2 n+2}$ (see Proposition 4.1). On applying Remark 4.3, we obtain that

$$
(Y) q=Y^{2 n^{2}} \mu_{2 n^{2}}+Y^{2 n^{2}-1} \mu_{2 n^{2}-1}+\ldots+Y \mu_{1}+E \mu_{0}=0 .
$$

Since $0 \neq \mu_{2 n^{2}} \in \mathbf{Z} \subset K$ and each $\mu_{i} \in G_{0}, 0 \leq i \leq 2 n^{2}$, is central, it follows that

$$
\left[Y^{2 n^{2}}, Z\right]=-\mu_{2 n^{2}-1} \mu_{2 n^{2}}^{-1}\left[Y^{2 n^{2}-1}, Z\right]-\ldots-\mu_{2} \mu_{2 n^{2}}^{-1}\left[Y^{2}, Z\right]-\mu_{1} \mu_{2 n^{2}}^{-1}[Y, Z]
$$

for all $Z \in M_{n}(G)$. Thus the multilinear and the alternating properties of the standard polynomial $S_{2 n^{2}}$ give the desired equality.

Remarks 5.2. (1) The equation $(Y) q=0$ in the above proof of Theorem 5.1 shows that $M_{n}(G)$ is integral of bounded degree over the commutative algebra $G_{0}$.

(2) The fact that $M_{n}(K)$ satisfies $S_{n}\left(\left[Y^{n}, Z\right],\left[Y^{n-1}, Z\right], \ldots,\left[Y^{2}, Z\right],[Y, Z]\right)=0$ is a classical theorem of Jacobson ([5]). 
(3) In view of recent results of Kantor and Trishin ([6]), one can expect that $S_{k}\left(\left[Y^{k}, Z\right],\left[Y^{k-1}, Z\right], \ldots,\left[Y^{2}, Z\right],[Y, Z]\right)=0$ is a polynomial identity for the matrix superalgebra

$$
M_{u, v}(G)=\left[\begin{array}{cc}
G_{0} & G_{1} \\
G_{1} & G_{0}
\end{array}\right]
$$

with $G_{0}$-blocks of sizes $u \times u, v \times v$ and $G_{1}$-blocks of sizes $u \times v, v \times u$, even if $k<2 n^{2}(u+v=n)$. Using the "relative" Cayley-Hamilton equation 4.17 or 4.19 in [6], one obtains that $S_{3}\left(\left[Y^{3}, Z\right],\left[Y^{2}, Z\right],[Y, Z]\right)=0$ is an identity for $M_{1,1}(G)$.

Theorem 5.3. Over an arbitrary field $K$, for any T-ideal $I$ of the free associative algebra $K\left\langle Y, Z, y_{1}, y_{2}, \ldots, y_{k}, \ldots\right\rangle$, there exists an integer $k \geq 1$ such that

$$
S_{k}\left(\left[Y^{k}, Z\right],\left[Y^{k-1}, Z\right], \ldots,\left[Y^{2}, Z\right],[Y, Z]\right) \in I .
$$

Proof. In characteristic zero Kemer proved that for some integer $n \geq 1$ the T-ideal of the identities of $M_{n}(G)$ is contained in $I$ (see p.20 in [9]) and thus Theorem 5.1 applies. If $\operatorname{char}(K)=p>0$ then $I$ contains a standard polynomial, as it was proved in $[8]$.

\section{ACKNOWLEDGEMENT}

The present form of Theorem 5.3 is due to the referee. The author is grateful to her/him for providing this result, which is much stronger than the original version was.

\section{REFERENCES}

1. A. Berele and A. Regev, Applications of hook Young diagrams to PI-algebras, J. Algebra 82 (1983), 559-567. MR 84g:16012

2. A. Berele, Magnum P.I., Israel J. Math. 51 (1985), 13-19. MR 87b:16019

3. A. Berele, Azumaya-like properties of verbally prime algebras, J. Algebra 133 No.2, (1990), 272-276. MR 91g:16014

4. B. Bódi and I.I. Khripta, Generalized Lie nilpotent group rings, Mat. Sbornik 129 No.1, (1986), 154-158. MR 87e:16031

5. N. Jacobson, Structure theory of algebraic algebras of bounded degree, Ann. of Math. 46 (1945), 695-707. MR 7:238c

6. I. Kantor and I. Trishin, On a concept of determinant in the supercase, Comm. Algebra 22 (10), (1994), 3679-3739. MR 95c:15062

7. A.R. Kemer, Varieties of $\mathbf{Z}_{2}$-graded algebras, Math. USSR Izv. 25 (1985), 359-374.

8. A.R. Kemer, The standard identity in characteristic $p$ : a conjecture of I. B. Volichenko, Israel J. Math. 8 (1993), 343-355. MR 94f: 16040

9. A.R. Kemer, Ideals of Identities of Associative Algebras, Translations of Math. Monographs, Vol.87 (1991), AMS Providence, Rhode Island. MR 92f:16031

10. D. Krakowsky and A. Regev, The polynomial identities of the Grassmann algebra, Trans. Amer. Math. Soc. 181 (1973), 429-438. MR 48:4005

11. I.B.S. Passi, D.S. Passman and S.K. Sehgal, Lie solvable group rings, Can. J. Math. 25 No. 4, (1973), 748-757. MR 48:4092

12. A. Popov, On the identities of the matrices over the Grassmann algebra, J. Algebra 168 No. 3, (1994), 828-852. MR 95h:16029

Institute of Mathematics, University of Miskold, Miskold-Egyetemváros, 3515 HunGARY

E-mail address: matszj@gold.uni-miskolc.hu 